\title{
Complex trapezoidal intuitionistic fuzzy numbers
}

\author{
R. Parvathi and J. Akila Padmasree \\ Department of Mathematics, Vellalar College for Women \\ Erode - 638 012, Tamilnadu, India \\ e-mails: paarvathis@rediffmail.com, \\ akilaa6666@gmail.com
}

Received: 16 May 2018

Accepted: 7 November 2018

\begin{abstract}
The paper introduces the Complex Trapezoidal Intuitionistic Fuzzy Numbers (CTrIFNs) in Cartesian form. Basic arithmetic operations are proposed based on $(\alpha, \beta)$ cuts and verified with suitable illustrations.
\end{abstract}

Keywords: Complex trapezoidal intuitionistic fuzzy number (CTrIFN), Trapezoidal intuitionistic fuzzy number (TrIFN).

2010 Mathematics Subject Classification: 03E72, 05C72, 05C65, 47N60.

\section{Introduction}

The real world situations are often associated with built-in uncertainities. The exact values are not known but the range of possible values are known. Uncertainities expressed in the form of intervals are apt in formulating the reality as a mathematical model which makes TrIFN a better option. CTrIFNs may be helpful in dealing uncertainities which are multi-dimensional in real life situations. The need for CTrIFNs arises when visualizing the areas where uncertainities are continuous and multi-dimensional. For example, turbulence is a violent or unsteady movement of air, water or some other fluid. Turbulence is chaotic and varies with time and direction. Uncertainities arise from the pressure difference at various points and also from the direction which depends on the fluctuations of the turbulence. These critical points are more clear when expressed using interval numbers. The critical points are dependant on multiple issues like magnitude, direction, pressure and the human perception. Complex numbers provide a better understanding of uncertainities with multi-dimensional quantities. Hence, the need for extension of Trapezoidal Intuitionistic 
Fuzzy Numbers into complex number system arises. Intuitionistic fuzzy sets were introduced by Krassimir T. Atanassov [1]. J. J. Buckley defined the Fuzzy Complex Numbers [3]. Burillo et al. proposed the defintion of intuitionistic fuzzy numbers [6]. The arithmetic operations on Trapezoidal Intuitionistic Fuzzy Numbers using $(\alpha, \beta)$ cuts were defined by Thangaraj Beaula and M. Priyadharsini [4]. R. Parvathi and C. Malathi developed arithmetic operations on Symmetric Trapezoidal Intuitionistic Fuzzy Numbers [2]. Moore [5] developed the interval arithmetic as a formal system. With all these motivations, Complex Trapezoidal Intuitionistic Fuzzy Numbers (CTrIFNs) are introduced in this paper.

\section{Notations and preliminaries}

We remind the reader that $Z=A+i B$ is a Complex Trapezoidal Intuitionistic Fuzzy Number, where $A=\left[a, b, c, d ; a^{\prime}, b, c, d^{\prime}\right]$ and $B=\left[p, q, r, s ; p^{\prime}, q, r, s^{\prime}\right]$ are TrIFNs such that $a^{\prime} \leq a \leq$ $b \leq c \leq d \leq d^{\prime}$. In this section, basic definitions relating to intuitionistic fuzzy sets, trapezoidal intuitionistic fuzzy numbers which are prerequisites for this study are dealt with.

Definition 2.1. [1] Let a set $X$ be fixed. An intuitionistic fuzzy set (IFS) $A$ in $X$ is an object of the form $A=\left\{\left\langle x, \mu_{A}(x), \nu_{A}(x)\right\rangle \mid x \in X\right\}$, where the function $\mu_{A}: X \rightarrow[0,1]$ and $\nu_{A}: X \rightarrow[0,1]$ determine the degree of membership and the degree of non-membership of the element $x \in X$, respectively and for every $x \in X, 0 \leq \mu_{A}(x)+\nu_{A}(x) \leq 1$ and $\pi_{A}(x)=1-\mu_{A}(x)-\nu_{A}(x)$ is the degree of uncertainity of $x$ in $A$, such that $0 \leq \pi_{A}(x) \leq 1$.

Definition 2.2. [6] An intuitionistic fuzzy number (IFN) $A$ is defined as follows:

- It is an intuitionistic fuzzy subset of the real line;

- It is normal, that is, there exists a $x \in R$ such that $\mu_{A}(x)=1$, (So, $\left.\nu_{A}(x)=\pi_{A(x)}=0\right)$;

- It is a convex set for the membership function $\mu_{A}(x)$. That is, $\mu_{A}\left(\lambda x_{1}+(1-\lambda) x_{2}\right) \geq$ $\min \left(\mu_{A}\left(x_{1}\right), \mu_{A}\left(x_{2}\right)\right)$ for all $x_{1}, x_{2} \in R$ and $\lambda \in[0,1]$;

- It is a concave set for the non-membership function $\nu_{A}(x)$. That is, $\nu_{A}\left(\lambda x_{1}+(1-\lambda) x_{2}\right) \geq$ $\max \left(\nu_{A}\left(x_{1}\right), \nu_{A}\left(x_{2}\right)\right)$ for all $x_{1}, x_{2} \in R$ and $\lambda \in[0,1]$.

Definition 2.3. [2] A Trapezoidal intuitionistic fuzzy number (TrIFN) $A$ is an intuitionistic fuzzy set in $R$ with the membership and non-membership fuctions as given below:

$$
\mu_{A}(x)=\left\{\begin{array}{ll}
\frac{x-\left(a_{1}-\alpha\right)}{\alpha} & x \in\left[a_{1}-\alpha, a_{1}\right] \\
1 & x \in\left[a_{1}, a_{2}\right] \\
\frac{a_{2}+\beta-x}{\beta} & x \in\left[a_{2}, a_{2}+\beta\right] \\
0 & \text { otherwise }
\end{array}, \quad \nu_{A}(x)= \begin{cases}\frac{a_{1}-x}{\alpha^{\prime}} & x \in\left[a_{1}-\alpha^{\prime}, a_{1}\right] \\
0 & x \in\left[a_{1}, a_{2}\right] \\
\frac{x-a_{2}}{\beta^{\prime}} & x \in\left[a_{2}, a_{2}+\beta^{\prime}\right] \\
1 & \text { otherwise }\end{cases}\right.
$$

where $a_{1} \leq a_{2}, \alpha, \beta \geq 0$ such that $\alpha \leq \alpha^{\prime}$ and $\beta \leq \beta^{\prime}$. A TrIFN is denoted by $A_{\operatorname{TrIFN}}=$ $\left[a_{1}, a_{2}, \alpha, \beta ; a_{1}, a_{2}, \alpha^{\prime}, \beta^{\prime}\right]$. 
Note: In Definition 2.3, the following changes in notations are made and used in this paper. When $a_{1}=b, a_{2}=c, a_{1}-\alpha=a, a_{2}+\beta=d, a_{1}-\alpha^{\prime}=a^{\prime}$ and $a_{2}+\beta^{\prime}=d^{\prime}$ in Definition 2.3, then the membership and non-membership functions take the form

$$
\mu_{A}(x)=\left\{\begin{array}{ll}
\frac{x-a}{b-a} & x \in[a, b] \\
1 & x \in[b, c] \\
\frac{d-x}{d-c} & x \in[c, d] \\
0 & \text { otherwise }
\end{array}, \quad \nu_{A}(x)=\left\{\begin{array}{ll}
\frac{b-x}{b-a^{\prime}} & x \in\left[a^{\prime}, b\right] \\
0 & x \in[b, c] \\
\frac{x-c}{d^{\prime}-c} & x \in\left[c, d^{\prime}\right] \\
1 & \text { otherwise }
\end{array},\right.\right.
$$

where $b \leq c$ and $(b-a) \geq 0$ which gives $b \geq a .(d-c) \geq 0$ which gives $d \geq c$. Therefore, $a \leq b \leq c \leq d$. Also, $(b-a) \leq b-a^{\prime}$. Therefore, $a^{\prime} \leq a$ and similarly, $d \leq d^{\prime}$. Hence, $a^{\prime} \leq a \leq b \leq c \leq d \leq d^{\prime}$. Thus $A_{T r I F N}=\left[a, b, c, d ; a^{\prime}, b, c, d^{\prime}\right]$.

Definition 2.4. [4] A $\alpha$-cut of $\operatorname{TrIFN} A=[a, b, c, d]$ is a crisp subset of $R$ and is defined as

$$
A_{\alpha}=\left\{x \mid \mu_{A}(x) \geq \alpha\right\} .
$$

Thus, $A_{\alpha}$ is a closed interval and is denoted by $A_{\alpha}=\left[L_{\alpha}(A), R_{\alpha}(A)\right]$.

Definition 2.5. [4] A $\beta$-cut of $\operatorname{TrIFN} A=[a, b, c, d]$ is a crisp subset of $R$ and is defined as

$$
A_{\beta}=\left\{x \mid \nu_{A}(x) \leq \beta\right\} .
$$

Thus, $A_{\beta}$ is a closed interval and is denoted by $A_{\beta}=\left[L_{\alpha}(A), R_{\alpha}(A)\right]$.

Assumptions: Let $R$ be the set of real numbers and $x \in R$. Let $a^{\prime}, a, b, c, d, d^{\prime}, p^{\prime}, p, q, r, s, s^{\prime} \in R$ such that $a^{\prime} \leq a \leq b \leq c \leq d \leq d^{\prime}$ and $p^{\prime} \leq p \leq q \leq r \leq s \leq s^{\prime}$. Let $A=\left[a, b, c, d ; a^{\prime}, b, c, d^{\prime}\right]$ and $B=\left[p, q, r, s ; p^{\prime}, q, r, s^{\prime}\right]$ be two trapezoidal intuitionistic fuzzy numbers (TrIFNs) in $R$ with membership values $\mu_{A}(x), \mu_{B}(x)$ and non-membership values $\nu_{A}(x), \nu_{B}(x)$ for every $x \in R$.

\section{Definition of CTrIFN}

Let $A$ and $B$ be two TrIFNs. A Complex Trapezoidal Intuitionistic Fuzzy Number (CTrIFN) $Z$ is a trapezoidal intuitionistic fuzzy number in the complex plane $C$ and is of the form $Z=\left\langle A+i B,\left(\mu_{A}, \nu_{A}\right),\left(\mu_{B}, \nu_{B}\right)\right\rangle$, where $\left(\mu_{A}, \nu_{A}\right)$ denotes the degrees of membership and nonmembership values of $\operatorname{Re}(Z)$ and $\left(\mu_{B}, \nu_{B}\right)$ denotes the degrees membership and non-membership values of $\operatorname{Im}(Z)$. In other words, $Z$ has the form $Z=\left[a, b, c, d ; a^{\prime}, b, c, d^{\prime}\right]+i\left[p, q, r, s ; p^{\prime}, q, r, s^{\prime}\right]$, where the membership and non-membership functions of $\operatorname{Re}(Z)$ and $\operatorname{Im}(Z)$ are defined as follows:

For every $x \in R$,

$$
\mu_{A}(x)=\left\{\begin{array}{ll}
\frac{x-a}{b-a} & a \leq x \leq b \\
1 & b \leq x \leq c \\
\frac{d-x}{d-c} & c \leq x \leq d \\
0 & \text { otherwise }
\end{array}, \quad \nu_{A}(x)= \begin{cases}\frac{b-x}{b-a^{\prime}} & a^{\prime} \leq x \leq b \\
0 & b \leq x \leq c \\
\frac{x-c}{d^{\prime}-c} & c \leq x \leq d^{\prime} \\
1 & \text { otherwise }\end{cases}\right.
$$




$$
\mu_{B}(x)=\left\{\begin{array}{ll}
\frac{x-p}{q-p} & p \leq x \leq q \\
1 & q \leq x \leq r \\
\frac{s-x}{s-r} & r \leq x \leq s \\
0 & \text { otherwise }
\end{array}, \quad \nu_{B}(x)= \begin{cases}\frac{q-x}{q-p^{\prime}} & p^{\prime} \leq x \leq q \\
0 & q \leq x \leq r \\
\frac{x-r}{s^{\prime}-r} & r \leq x \leq s^{\prime} \\
1 & \text { otherwise }\end{cases}\right.
$$

where $a^{\prime} \leq a \leq b \leq c \leq d \leq d^{\prime}$ and $a^{\prime}, a, b, c, d, d^{\prime} \in R .0 \leq \mu_{A} \leq 1$ and $0 \leq \nu_{A} \leq 1$ and $0 \leq \mu_{A}+\nu_{A} \leq 1$. Also, $p^{\prime} \leq p \leq q \leq r \leq s \leq s^{\prime}$ and $p^{\prime}, p, q, r, s, s^{\prime} \in R$. $0 \leq \mu_{B} \leq 1$,

$0 \leq \nu_{B} \leq 1$ and $0 \leq \mu_{B}+\nu_{B} \leq 1$. For simplicity, we write $Z=\left\langle[a, b, c, d] ; \mu_{A}, \nu_{A}\right\rangle+$ $i\left\langle[p, q, r, s] ; \mu_{B}, \nu_{B}\right\rangle$.

Remark: A CTrIFN becomes a CTrFN by letting $a=a^{\prime}$ and $d=d^{\prime}$ and $\nu_{A}=1-\mu_{A}$.

\section{Arithmetic operations on CTrIFNs}

\subsection{Addition}

If $Z_{1}=A+i B$ and $Z_{2}=C+i D$ are two CTrIFNs, then $Z=Z_{1}+Z_{2}=(A+C)+i(B+D)$ is also a CTrIFN. $Z$ takes the form

$$
\begin{aligned}
Z=\left[a+a_{1}, b+b_{1}, c+c_{1},\right. & \left.d+d_{1} ; a^{\prime}+a_{1}^{\prime}, b+b_{1}, c+c_{1}, d^{\prime}+d_{1}^{\prime}\right] \\
+ & i\left[p+p_{1}, q+q_{1}, r+r_{1}, s+s_{1} ; p^{\prime}+p_{1}^{\prime}, q+q_{1}, r+r_{1}, s^{\prime}+s_{1}^{\prime}\right]
\end{aligned}
$$

with the membership and non-membership values of $\operatorname{Re}(Z)$ and $\operatorname{Im}(Z)$, for every $x \in R$ as

$$
\begin{aligned}
& \mu_{A+C}(x)= \begin{cases}\frac{x-a-a_{1}}{(b-a)+\left(b_{1}-a_{1}\right)} & a+a_{1} \leq x \leq b+b_{1} \\
1 & b+b_{1} \leq x \leq c+c_{1} \\
\frac{d+d_{1}-x}{(d-c)+\left(d_{1}-c_{1}\right)} & c+c_{1} \leq x \leq d+d_{1} \\
0 & \text { otherwise }\end{cases} \\
& \nu_{A+C}(x)= \begin{cases}\frac{b+b_{1}-x}{\left(b-a^{\prime}\right)+\left(b_{1}-a_{1}^{\prime}\right)} & a^{\prime}+a_{1}^{\prime} \leq x \leq b+b_{1} \\
0 & b+b_{1} \leq x \leq c+c_{1} \\
\frac{x-c-c_{1}}{\left(d^{\prime}-c\right)+\left(d_{1}^{\prime}-c_{1}\right)} & c+c_{1} \leq x \leq d^{\prime}+d_{1}^{\prime} \\
1 & \text { otherwise }\end{cases} \\
& \mu_{B+D}(x)= \begin{cases}\frac{x-p-p_{1}}{(q-p)+\left(q_{1}-p_{1}\right)} & p+p_{1} \leq x \leq q+q_{1} \\
1 & q+q_{1} \leq x \leq r+r_{1} \\
\frac{s+s_{1}-x}{(s-r)+\left(s_{1}-r_{1}\right)} & r+r_{1} \leq x \leq s+s_{1} \\
0 & \text { otherwise }\end{cases}
\end{aligned}
$$




$$
\nu_{B+D}(x)= \begin{cases}\frac{q+q_{1}-x}{\left(q-p^{\prime}\right)+\left(q_{1}-p_{1}^{\prime}\right)} & p^{\prime}+p_{1}^{\prime} \leq x \leq q+q_{1} \\ 0 & q+q_{1} \leq x \leq r+r_{1} \\ \frac{x-r-r_{1}}{\left(s^{\prime}-r\right)+\left(s_{1}^{\prime}-r_{1}\right)} & r+r_{1} \leq x \leq s^{\prime}+s_{1}^{\prime} \\ 1 & \text { otherwise }\end{cases}
$$

Proof. The membership and non-membership functions of CTrIFN $Z=(A+C)+i(B+D)$ can be found by $(\alpha, \beta)$ cuts. Now, $\alpha$-cut of $A$ is $[a+\alpha(b-a), d-\alpha(d-c)]$ and $\alpha$-cut of $C$ is $\left[a_{1}+\alpha\left(b_{1}-a_{1}\right), d_{1}-\alpha\left(d_{1}-c_{1}\right)\right]$. Therefore, $\alpha$-cut of $(A+C)$ is

$$
\alpha_{A}+\alpha_{C}=\left[a+a_{1}+\alpha\left(b-a+b_{1}-a_{1}\right), d+d_{1}-\alpha\left(d-c+d_{1}-c_{1}\right)\right] .
$$

Equating the components to $x$ and expressing in terms of $\alpha$ gives the membership function of $\operatorname{Re}(Z)$ as,

$$
\mu_{A+C}(x)= \begin{cases}\frac{x-a-a_{1}}{(b-a)+\left(b_{1}-a_{1}\right)} & a+a_{1} \leq x \leq b+b_{1} \\ 1 & b+b_{1} \leq x \leq c+c_{1} \\ \frac{d+d_{1}-x}{(d-c)+\left(d_{1}-c_{1}\right)} & c+c_{1} \leq x \leq d+d_{1} \\ 0 & \text { otherwise }\end{cases}
$$

Now, $\beta$-cut of $A$ is $\left[b-\beta\left(b-a^{\prime}\right), c+\beta\left(d^{\prime}-c\right)\right]$ and $\beta$-cut of $C$ is $\left[b_{1}-\beta\left(b_{1}-a_{1}^{\prime}\right), \beta\left(d_{1}^{\prime}-c_{1}\right)+c\right]$. Therefore, $\beta$-cut of $A+C$ is $\left[b+b_{1}-\beta\left(b-a^{\prime}+b_{1}-a_{1}^{\prime}\right), c+c_{1}+\beta\left(d^{\prime}-c-c_{1}+d_{1}^{\prime}\right)\right]$. Equating both the components to $x$ and expressing in terms of $\beta$ gives the non-membership function of $\operatorname{Re}(Z)$ as,

$$
\nu_{A+C}(x)= \begin{cases}\frac{b+b_{1}-x}{\left(b-a^{\prime}\right)+\left(b_{1}-a_{1}^{\prime}\right)} & a^{\prime}+a_{1}^{\prime} \leq x \leq b+b_{1} \\ 0 & b+b_{1} \leq x \leq c+c_{1} \\ \frac{x-c-c_{1}}{d^{\prime}-c-c_{1}+d_{1}^{\prime}} & c+c_{1} \leq x \leq d^{\prime}+d_{1}^{\prime} \\ 1 & \text { otherwise }\end{cases}
$$

Now, the sum of the $\alpha$-cut of $B$ and the $\alpha$-cut of $D$ is $[p+\alpha(q-p), s-\alpha(s-r)]+$ $\alpha\left(q_{1}-p_{1}\right)+p_{1}, s_{1}-\alpha\left(s_{1}-r_{1}\right)=\left[p+p_{1}+\alpha\left(q-p+q_{1}-p_{1}\right), s+s_{1}-\alpha\left(s-r+s_{1}-r_{1}\right)\right]$.

Equating each term to $x$ gives the membership function of $\operatorname{Im}(Z)$ as,

$$
\mu_{B+D}(x)= \begin{cases}\frac{x-p-p_{1}}{(q-p)+\left(q_{1}-p_{1}\right)} & p+p_{1} \leq x \leq q+q_{1} \\ 1 & q+q_{1} \leq x \leq r+r_{1} \\ \frac{s+s_{1}-x}{(s-r)+\left(s_{1}-r_{1}\right)} & r+r_{1} \leq x \leq s+s_{1} \\ 0 & \text { otherwise }\end{cases}
$$

Here, the sum of the $\beta$-cut of $B$ and the $\beta$-cut of $D$ is

$$
\left[q+q_{1}-\beta\left(q-p^{\prime}+q_{1}-p_{1}^{\prime}\right), r+r_{1}+\beta\left(s^{\prime}-r+s_{1}^{\prime}-r_{1}\right)\right] .
$$


Equating each term to $x$ gives the non-membership function of $\operatorname{Im}(Z)$ as,

$$
\nu_{B+D}(x)= \begin{cases}\frac{q+q_{1}-x}{\left(q-p^{\prime}\right)+\left(q_{1}-p_{1}^{\prime}\right)} & p^{\prime}+p_{1}^{\prime} \leq x \leq q+q_{1} \\ 0 & q+q_{1} \leq x \leq r+r_{1} \\ \frac{x-r-r_{1}}{\left(s^{\prime}-r\right)-r_{1}+s_{1}^{\prime}} & r+r_{1} \leq x \leq s^{\prime}+s_{1}^{\prime} \\ 1 & \text { otherwise }\end{cases}
$$

\subsection{Subtraction}

Consider the two TrIFNs $Z_{1}$ and $Z_{2}$ such that $Z_{1}=A+i B$ and $Z_{2}=C+i D$. The difference of two CTrIFNs denoted by $Z_{1}-Z_{2}$ is defined as $Z=Z_{1}-Z_{2}=(A-C)+i(B-D)$, where, $\mu_{A-C}$ and $\nu_{A-C}$ are membership and non-membership functions of the real part and $\mu_{B-D}$ and $\nu_{B-D}$ are membership and non-membership functions of the imaginary part. $Z$ takes the form

$$
\begin{aligned}
& Z=\left[a-d_{1}, b-c_{1}, c-b_{1},\right.\left.d-a_{1} ; a^{\prime}-d_{1}^{\prime}, b-c_{1}, c-b_{1}, d^{\prime}-a_{1}^{\prime}\right] \\
&+i\left[p-s_{1}, q-r_{1}, r-q_{1}, s-p_{1} ; p^{\prime}-s_{1}^{\prime}, q-r_{1}, r-q_{1}, s^{\prime}-p_{1}^{\prime}\right]
\end{aligned}
$$

with the membership and non-membership values of $\operatorname{Re}(Z)$ and $\operatorname{Im}(Z)$ as,

$$
\begin{aligned}
& \mu_{A-C}(x)= \begin{cases}\frac{x-a+d_{1}}{(b-a)+\left(d_{1}-c_{1}\right)} & a-d_{1} \leq x \leq b-c_{1} \\
1 & b-c_{1} \leq x \leq c-b_{1} \\
\frac{d-a_{1}-x}{\left(b_{1}-a_{1}\right)+(d-c)} & c-b_{1} \leq x \leq d-a_{1} \\
0 & \text { otherwise }\end{cases} \\
& \nu_{A-C}(x)= \begin{cases}\frac{b+c_{1}-x}{\left(b-a^{\prime}\right)+\left(d_{1}^{\prime}-c_{1}\right)} & a^{\prime}-d_{1}^{\prime} \leq x \leq b-c_{1} \\
0 & b-c_{1} \leq x \leq c-b_{1} \\
\frac{x-c+b_{1}}{\left(d^{\prime}-c\right)+\left(b_{1}-a_{1}^{\prime}\right)} & c-b_{1} \leq x \leq d^{\prime}-a_{1}^{\prime} \\
1 & \text { otherwise }\end{cases} \\
& \mu_{B-D}(x)= \begin{cases}\frac{x-p-s_{1}}{(q-p)+\left(s_{1}-r_{1}\right)} & p-s_{1} \leq x \leq q-r_{1} \\
1 & q-r_{1} \leq x \leq r-q_{1} \\
\frac{s-p_{1}-x}{(s-r)+\left(q_{1}-p_{1}\right)} & r-q_{1} \leq x \leq s-p_{1} \\
0 & \text { otherwise }\end{cases} \\
& \nu_{B-D}(x)= \begin{cases}\frac{q+r_{1}-x}{\left(b-a^{\prime}\right)+\left(d_{1}^{\prime}-c_{1}\right)} & p^{\prime}-s_{1}^{\prime} \leq x \leq q-r_{1} \\
0 & q-r_{1} \leq x \leq r-q_{1} \\
\frac{x-r+q_{1}}{\left(s^{\prime}-r\right)+\left(q_{1}-p_{1}^{\prime}\right)} & r-q_{1} \leq x \leq s^{\prime}-p_{1}^{\prime} \\
1 & \text { otherwise }\end{cases}
\end{aligned}
$$

Proof. The membership and non-membership functions of CTrIFN $Z=(A-C)+i(B-D)$ can be found by the $(\alpha, \beta)$ cuts. Now, the $\alpha$-cut of $A$ minus the $\alpha$-cut of $C$ is 


$$
\begin{aligned}
& {[(b-a) \alpha+a, d-(d-c) \alpha]-\left[a_{1}+\alpha\left(b_{1}-a_{1}\right), d_{1}-\alpha\left(d_{1}-c_{1}\right)\right]} \\
& =\left[\alpha\left(b-a+d_{1}-c_{1}\right)+\left(a-d_{1}\right),\left(d-a_{1}\right)-\alpha\left(b_{1}-a_{1}+d-c\right)\right] .
\end{aligned}
$$

Equating each term to $x$ gives the membership function of $\operatorname{Re}(Z)$ as,

$$
\mu_{A-C}(x)= \begin{cases}\frac{x-a+d_{1}}{(b-a)+\left(d_{1}-c_{1}\right)} & a-d_{1} \leq x \leq b-c_{1} \\ 1 & b-c_{1} \leq x \leq c-b_{1} \\ \frac{d-a_{1}-x}{\left(b_{1}-a_{1}\right)+(d-c)} & c-b_{1} \leq x \leq d-a_{1} \\ 0 & \text { otherwise }\end{cases}
$$

Now, the $\beta$-cut of $A$ minus the $\beta$-cut of $C$ is

$$
\left[\left(b+c_{1}\right)-\beta\left[\left(b-a^{\prime}\right)+\left(d_{1}^{\prime}-c_{1}\right)\right], \beta\left[\left(d^{\prime}-c\right)+\left(b_{1}-a_{1}^{\prime}\right)\right]+\left(c-b_{1}\right)\right]
$$

and equating each term to $x$ gives the non-membership function of $\operatorname{Re}(Z)$ as,

$$
\nu_{A-C}(x)= \begin{cases}\frac{b+c_{1}-x}{\left(b-a^{\prime}\right)+\left(d_{1}^{\prime}-c_{1}\right)} & a^{\prime}-d_{1}^{\prime} \leq x \leq b-c_{1} \\ 0 & b-c_{1} \leq x \leq c-b_{1} \\ \frac{x-c+b_{1}}{\left(d^{\prime}-c\right)+\left(b_{1}-a_{1}^{\prime}\right)} & c-b_{1} \leq x \leq d^{\prime}-a_{1}^{\prime} \\ 1 & \text { otherwise }\end{cases}
$$

Here, $\alpha$-cut of B - $\alpha$-cut of $D=\left[\alpha\left(q-p+s_{1}-r_{1}\right)+p-s_{1},\left(s-p_{1}\right)-\alpha\left(s-r+q_{1}-p_{1}\right)\right]$ and equating each term to $x$ gives the membership function of $\operatorname{Im}(Z)$ as,

$$
\mu_{B-D}(x)= \begin{cases}\frac{x-p-s_{1}}{(q-p)+\left(s_{1}-r_{1}\right)} & p-s_{1} \leq x \leq q-r_{1} \\ 1 & q-r_{1} \leq x \leq r-q_{1} \\ \frac{s-p_{1}-x}{(s-r)+\left(q_{1}-p_{1}\right)} & r-q_{1} \leq x \leq s-p_{1} \\ 0 & \text { otherwise }\end{cases}
$$

Similarly,

$$
\nu_{B-D}(x)= \begin{cases}\frac{q+r_{1}-x}{\left(b-a^{\prime}\right)+\left(d_{1}^{\prime}-c_{1}\right)} & p^{\prime}-s_{1}^{\prime} \leq x \leq q-r_{1} \\ 0 & q-r_{1} \leq x \leq r-q_{1} \\ \frac{x-r+q_{1}}{s^{\prime}-r+q_{1}-p_{1}^{\prime}} & r-q_{1} \leq x \leq s^{\prime}-p_{1}^{\prime} \\ 1 & \text { otherwise }\end{cases}
$$

\subsection{Product of CTrIFNS}

Consider the two TrIFNs $Z_{1}$ and $Z_{2}$ such that $Z_{1}=A+i B$ and $Z_{2}=C+i D$ with the membership values $\mu_{A}(x), \mu_{B}(x), \mu_{C}(x), \mu_{D}(x)$ and non-membership values $\nu_{A}(x), \nu_{B}(x), \nu_{C}(x), \nu_{D}(x)$. The product of two CTrIFNs denoted by $Z_{1} \cdot Z_{2}$ is defined as $Z=Z_{1} \cdot Z_{2}=(A C-B D)+$ $i(A D+B C)$, where, $\mu_{(A C-B D)}, \nu_{(A C-B D)}$ are the membership and non-membership values of 
the real part and $\mu_{(A D+B C)}, \nu_{(A D+B C)}$ are the membership and non-membership values of the imaginary part and $Z$ takes the form

$$
\begin{aligned}
& Z_{1} . Z_{2}=\left[a a_{1}-s s_{1}, b b_{1}-r r_{1}, c c_{1}-q q_{1}, d d_{1}-p p_{1} ; a^{\prime} a_{1}^{\prime}-s^{\prime} s_{1}^{\prime}, b b_{1}-r r_{1}, c c_{1}-q q_{1}, d^{\prime} d_{1}^{\prime}-p^{\prime} p_{1}^{\prime}\right] \\
& \quad+i\left[a p_{1}+p a_{1}, b q_{1}+q b_{1}, c r_{1}+r c_{1}, d s_{1}+s d_{1} ; a^{\prime} p_{1}^{\prime}+p^{\prime} a_{1}^{\prime}, b q_{1}+q b_{1}, c r_{1}+r c_{1}, d^{\prime} s_{1}^{\prime}+s^{\prime} d_{1}^{\prime}\right]
\end{aligned}
$$

with the membership and non-membership values of $\operatorname{Re}(Z)$ and $\operatorname{Im}(Z)$ as,

$$
\mu_{A C-B D}(x)= \begin{cases}\frac{-B_{1}+\sqrt{B_{1}^{2}-4 A_{1} C_{1}}}{2 A_{1}} & a a_{1}-s s_{1} \leq x \leq b b_{1}-r r_{1} \\ 1 & b b_{1}-r r_{1} \leq x \leq c c_{1}-q q_{1} \\ \frac{-B_{2}+\sqrt{B_{2}^{2}-4 A_{2} C_{2}}}{2 A_{2}} & c c_{1}-q q_{1} \leq x \leq d d_{1}-p p_{1} \\ 0 & \text { otherwise }\end{cases}
$$

where

$$
\begin{gathered}
B_{1}=\left[a_{1}(b-a)+a\left(b_{1}-a_{1}\right)+s\left(s_{1}-r_{1}\right)+s_{1}(s-r)\right] \\
A_{1}=\left[(b-a)\left(b_{1}-a_{1}\right)-(s-r)\left(s_{1}-r_{1}\right)\right] ; \\
C_{1}=\left(a a_{1}-s s_{1}-x\right) ; \\
B_{2}=\left[d\left(d_{1}-c_{1}\right)+d_{1}(d-c)+p_{1}(q-p)+p\left(q_{1}-p_{1}\right)\right] ; \\
A_{2}=\left[(d-c)\left(d_{1}-c_{1}\right)-(q-p)\left(q_{1}-p_{1}\right)\right] ; \\
\nu_{A C-B D}(x)= \begin{cases}\frac{B_{3}+\sqrt{B_{3}^{2}-4 A_{3} C_{3}}}{2 A_{3}} & a^{\prime} a_{1}^{\prime}-s^{\prime} s_{1}^{\prime} \leq x \leq b b_{1}-r r_{1} \\
0 & b b_{1}-r r_{1} \leq x \leq c c_{1}-q q_{1} \\
\frac{B_{4}+\sqrt{B_{4}^{2}-4 A_{4} C_{4}}}{2 A_{4}} & c c_{1}-q q_{1} \leq x \leq d^{\prime} d_{1}^{\prime}-p^{\prime} p_{1}^{\prime} \\
1 & \text { otherwise }\end{cases}
\end{gathered}
$$

where

$$
\begin{gathered}
B_{3}=\left[b\left(b_{1}-a_{1}^{\prime}\right)+b_{1}\left(b-a^{\prime}\right)+r_{1}\left(s^{\prime}-r\right)+r\left(s^{\prime}-r_{1}\right)\right] ; \\
A_{3}=\left[\left(b-a^{\prime}\right)\left(b_{1}-a_{1}^{\prime}\right)-\left(s^{\prime}-r\right)\left(s^{\prime}-r_{1}\right)\right] ; \\
C_{3}=\left(b b_{1}-r r_{1}-x\right) ; \\
B_{4}=\left[c_{1}\left(d^{\prime}-c\right)+c\left(d_{1}^{\prime}-c_{1}\right)+q_{1}\left(q_{1}-p_{1}^{\prime}\right)+q\left(q-p^{\prime}\right)\right] ; \\
A_{4}=\left[\left(d^{\prime}-c\right)\left(d_{1}^{\prime}-c\right)-\left(q-p^{\prime}\right)\left(q_{1}-p_{1}^{\prime}\right)\right] ; \\
\mu_{A D+B C}(x)= \begin{cases}\frac{-B_{1}+\sqrt{B_{1}^{2}-4 A_{1} C_{1}}}{2 A_{1}} & a p_{1}+p a_{1} \leq x \leq b q_{1}+q b_{1} \\
1 & b q_{1}+q b_{1} \leq x \leq c r_{1}+r c_{1} \\
\frac{-B_{2}+\sqrt{B_{2}^{2}-4 A_{2} C_{2}}}{2 A_{2}} & c r_{1}+r c_{1} \leq x \leq d s_{1}+s d_{1} \\
0 & \text { otherwise }\end{cases}
\end{gathered}
$$


where

$$
\begin{gathered}
B_{1}=\left[p_{1}(b-a)+a\left(q_{1}-p_{1}\right)+p\left(b_{1}-a_{1}\right)+a_{1}(q-p)\right] ; \\
A_{1}=\left[(b-a)\left(q_{1}-p_{1}\right)-(q-p)\left(b_{1}-a_{1}\right)\right] ; \\
C_{1}=\left(a p_{1}+p a_{1}-x\right) ; \\
B_{2}=\left[d\left(s_{1}-r_{1}\right)+s_{1}(d-c)+d_{1}(s-r)+s\left(d_{1}-c_{1}\right)\right] ; \\
A_{2}=\left[(d-c)\left(s_{1}-r_{1}\right)+(s-r)\left(d_{1}-c_{1}\right)\right] ; \\
\nu_{A D+B C}(x)= \begin{cases}\frac{B_{3}+\sqrt{B_{3}^{2}-4 A_{3} C_{3}}}{2 A_{3}} & a^{\prime} p_{1}^{\prime}+p^{\prime} a_{1}^{\prime} \leq x \leq b q_{1}+q b_{1} \\
0 & b q_{1}+q b_{1} \leq x \leq c r_{1}+r c_{1} \\
\frac{B_{4}+\sqrt{B_{4}^{2}-4 A_{4} C_{4}}}{2 A_{4}} & c r_{1}+r c_{1} \leq x \leq d^{\prime} s_{1}^{\prime}-s^{\prime} d_{1}^{\prime} \\
1 & \text { otherwise }\end{cases}
\end{gathered}
$$

where

$$
\begin{gathered}
B_{3}=\left[b\left(q_{1}-p_{1}^{\prime}\right)+q_{1}\left(b-a^{\prime}\right)+q\left(b_{1}-a_{1}^{\prime}\right)+b_{1}\left(q-p^{\prime}\right)\right] ; \\
A_{3}=\left[\left(b-a^{\prime}\right)\left(q_{1}-p_{1}^{\prime}\right)-\left(q-p^{\prime}\right)\left(b_{1}-a_{1}^{\prime}\right)\right] ; \\
C_{3}=\left(b q_{1}+q b_{1}-x\right) ; \\
B_{4}=\left[r_{1}\left(d^{\prime}-c\right)+c\left(s_{1}^{\prime}-r_{1}\right)+c_{1}\left(s^{\prime}-r\right)+r\left(d_{1}^{\prime}-c_{1}\right)\right] ; \\
A_{4}=\left[\left(d^{\prime}-c\right)\left(s_{1}^{\prime}-r_{1}\right)-\left(s^{\prime}-r\right)\left(d_{1}^{\prime}-c_{1}\right)\right] ; \\
C_{4}=\left(c r_{1}+r c_{1}-x\right) .
\end{gathered}
$$

The membership and non-membership functions are found using $(\alpha, \beta)$ cuts. Now, finding the $\alpha$-cut of $A C$ minus the $\alpha$-cut of $B D$ and equating each term to $x$ gives

$$
\begin{aligned}
x & =\alpha^{2}\left[(b-a)\left(b_{1}-a_{1}\right)-(s-r)\left(s_{1}-r_{1}\right)\right] \\
& +\alpha\left[a_{1}(b-a)+a\left(b_{1}-a_{1}\right)+s\left(s_{1}-r_{1}\right)+s_{1}(s-r)\right]+\left(a a_{1}-s s_{1}\right) \\
x & =\alpha^{2}\left[(d-c)\left(d_{1}-c_{1}\right)-(q-p)\left(q_{1}-p_{1}\right)\right] \\
& -\alpha\left[d\left(d_{1}-c_{1}\right)+d_{1}(d-c)+p_{1}(q-p)+p\left(q_{1}-p_{1}\right)\right]+\left(d d_{1}-p p_{1}\right) .
\end{aligned}
$$

The membership function of $\operatorname{Re}(Z)$ is,

$$
\mu_{A C-B D}(x)= \begin{cases}\frac{-B_{1}+\sqrt{B_{1}^{2}-4 A_{1} C_{1}}}{2 A_{1}} & a a_{1}-s s_{1} \leq x \leq b b_{1}-r r_{1} \\ 1 & b b_{1}-r r_{1} \leq x \leq c c_{1}-q q_{1} \\ \frac{-B_{2}+\sqrt{B_{2}^{2}-4 A_{2} C_{2}}}{2 A_{2}} & c c_{1}-q q_{1} \leq x \leq d d_{1}-p p_{1} \\ 0 & \text { otherwise }\end{cases}
$$

where

$$
B_{1}=\left[a_{1}(b-a)+a\left(b_{1}-a_{1}\right)+s\left(s_{1}-r_{1}\right)+s_{1}(s-r)\right]
$$




$$
\begin{gathered}
A_{1}=\left[(b-a)\left(b_{1}-a_{1}\right)-(s-r)\left(s_{1}-r_{1}\right)\right] ; \\
C_{1}=\left(a a_{1}-s s_{1}-x\right) ; \\
B_{2}=\left[d\left(d_{1}-c_{1}\right)+d_{1}(d-c)+p_{1}(q-p)+p\left(q_{1}-p_{1}\right)\right] ; \\
A_{2}=\left[(d-c)\left(d_{1}-c_{1}\right)-(q-p)\left(q_{1}-p_{1}\right)\right] ; \\
C_{2}=\left(d d_{1}-p p_{1}-x\right) .
\end{gathered}
$$

Similarly, the non-membership function of $\operatorname{Re}(Z)$,

$$
\nu_{A C-B D}(x)= \begin{cases}\frac{B_{3}+\sqrt{B_{3}^{2}-4 A_{3} C_{3}}}{2 A_{3}} & a^{\prime} a_{1}^{\prime}-s^{\prime} s_{1}^{\prime} \leq x \leq b^{\prime} b_{1}^{\prime}-r^{\prime} r_{1}^{\prime} \\ 0 & b^{\prime} b_{1}^{\prime}-r^{\prime} r_{1}^{\prime} \leq x \leq c^{\prime} c_{1}^{\prime}-q^{\prime} q_{1}^{\prime} \\ \frac{B_{4}+\sqrt{B_{4}^{2}-4 A_{4} C_{4}}}{2 A_{4}} & c^{\prime} c_{1}^{\prime}-q^{\prime} q_{1}^{\prime} \leq x \leq d^{\prime} d_{1}^{\prime}-p^{\prime} p_{1}^{\prime} \\ 1 & \text { otherwise }\end{cases}
$$

where

$$
\begin{gathered}
B_{3}=\left[b\left(b_{1}-a_{1}^{\prime}\right)+b_{1}\left(b-a^{\prime}\right)+r_{1}\left(s^{\prime}-r\right)+r\left(s^{\prime}-r_{1}\right)\right] ; \\
A_{3}=\left[\left(b-a^{\prime}\right)\left(b_{1}-a_{1}^{\prime}\right)-\left(s^{\prime}-r\right)\left(s^{\prime}-r_{1}\right)\right] ; \\
C_{3}=\left(b b_{1}-r r_{1}-x\right) ; \\
B_{4}=\left[c_{1}\left(d^{\prime}-c\right)+c\left(d_{1}^{\prime}-c_{1}\right)+q_{1}\left(q_{1}-p_{1}^{\prime}\right)+q\left(q-p^{\prime}\right)\right] ; \\
A_{4}=\left[\left(d^{\prime}-c\right)\left(d_{1}^{\prime}-c\right)-\left(q-p^{\prime}\right)\left(q_{1}-p_{1}^{\prime}\right)\right] ; \\
C_{4}=\left(c c_{1}-q q_{1}-x\right) .
\end{gathered}
$$

Similarly, the membership function of $\operatorname{Im}(Z)$,

$$
\mu_{A D+B C}(x)= \begin{cases}\frac{-B_{1}+\sqrt{B_{1}^{2}-4 A_{1} C_{1}}}{2 A_{1}} & a p_{1}+p a_{1} \leq x \leq b q_{1}+q b_{1} \\ 1 & b q_{1}+q b_{1} \leq x \leq c r_{1}+r c_{1} \\ \frac{-B_{2}+\sqrt{B_{2}^{2}-4 A_{2} C_{2}}}{2 A_{2}} & c r_{1}+r c_{1} \leq x \leq d s_{1}+s d_{1} \\ 0 & \text { otherwise }\end{cases}
$$

where

$$
\begin{gathered}
B_{1}=\left[p_{1}(b-a)+a\left(q_{1}-p_{1}\right)+p\left(b_{1}-a_{1}\right)+a_{1}(q-p)\right] ; \\
A_{1}=\left[(b-a)\left(q_{1}-p_{1}\right)-(q-p)\left(b_{1}-a_{1}\right)\right] ; \\
C_{1}=\left(a p_{1}+p a_{1}-x\right) ; \\
B_{2}=\left[d\left(s_{1}-r_{1}\right)+s_{1}(d-c)+d_{1}(s-r)+s\left(d_{1}-c_{1}\right)\right] ; \\
A_{2}=\left[(d-c)\left(s_{1}-r_{1}\right)+(s-r)\left(d_{1}-c_{1}\right)\right] ; \\
C_{2}=\left(d s_{1}+s d_{1}-x\right) .
\end{gathered}
$$


Similarly, the non-membership function of $\operatorname{Im}(Z)$

$$
\nu_{A D+B C}(x)= \begin{cases}\frac{B_{3}+\sqrt{B_{3}^{2}-4 A_{3} C_{3}}}{2 A_{3}} & a^{\prime} p_{1}^{\prime}+p^{\prime} a_{1}^{\prime} \leq x \leq b q_{1}+q b_{1} \\ 0 & b q_{1}+q b_{1} \leq x \leq c r_{1}+r c_{1} \\ \frac{B_{4}+\sqrt{B_{4}^{2}-4 A_{4} C_{4}}}{2 A_{4}} & c r_{1}+r c_{1} \leq x \leq d^{\prime} s_{1}^{\prime}-s^{\prime} d_{1}^{\prime} \\ 1 & \text { otherwise }\end{cases}
$$

where

$$
\begin{gathered}
B_{3}=\left[b\left(q_{1}-p_{1}^{\prime}\right)+q_{1}\left(b-a^{\prime}\right)+q\left(b_{1}-a_{1}^{\prime}\right)+b_{1}\left(q-p^{\prime}\right)\right] ; \\
A_{3}=\left[\left(b-a^{\prime}\right)\left(q_{1}-p_{1}^{\prime}\right)-\left(q-p^{\prime}\right)\left(b_{1}-a_{1}^{\prime}\right)\right] ; \\
C_{3}=\left(b q_{1}+q b_{1}-x\right) ; \\
B_{4}=\left[r_{1}\left(d^{\prime}-c\right)+c\left(s_{1}^{\prime}-r_{1}\right)+c_{1}\left(s^{\prime}-r\right)+r\left(d_{1}^{\prime}-c_{1}\right)\right] ; \\
A_{4}=\left[\left(d^{\prime}-c\right)\left(s_{1}^{\prime}-r_{1}\right)-\left(s^{\prime}-r\right)\left(d_{1}^{\prime}-c_{1}\right)\right] ; \\
C_{4}=\left(c r_{1}+r c_{1}-x\right) .
\end{gathered}
$$

\subsection{Scalar multiplication}

Let $Z=A+i B$ be a CTrIFN and $k$ is a scalar then

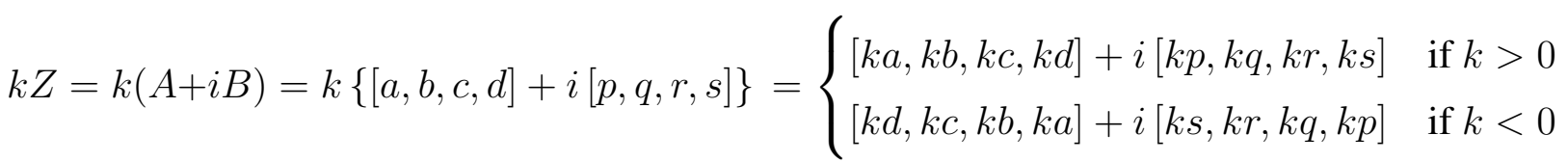

Definition 4.1. For any CTrIFN $Z=A+i B$, the negation is formulated as $-Z=-A-i B$.

Definition 4.2. For any CTrIFN $Z=A+i B$, the conjugate is given by $Z=A+i(-B)$.

\subsection{Important properties of CTrIFNS}

\subsubsection{Additive inverse does not exist for CTrIFN}

For, let $Z=A+i B$. Therefore, $-Z=-[A+i B]=-A-i B=-A+i(-B)=$ $[-d,-c,-b,-a]+i[-s,-r,-q,-p]$. This implies $Z+(-Z)=[a, b, c, d]+i[p, q, r, s]+$ $[-d,-c,-b,-a]+i[-s,-r,-q,-p]=[a-d, b-c, c-b, d-a]+i[p-s, q-r, r-q, s-p]$ $\neq 0$.

Therefore, the additive inverse does not exist for a CTrIFn.

Note: The additive inverse for a CTrIFN exists only if the interval is degenerate. That is, $a=b=$ $c=d$ and $p=q=r=s$. In this case, it is a complex number. 


\subsubsection{Additive property of a conjugate of CTrIFN}

The additive property of a conjugate of a CTrIFN does not hold, that is $(A+i B)+(A-i B) \neq 2 A$.

Proof. $(A+i B)+(A-i B)=(A+A)+i(B-B)=2 A+i(B-B)$

$=2[a, b, c, d]+i\langle[p, q, r, s]-[p, q, r, s]\rangle=2[a, b, c, d]+i\langle[p-s, q-r, r-q, s-p]\rangle$

$\neq 2 A$ (because of the lack of additive inverse).

\subsubsection{Multiplicative property of a conjugate of CTrIFN}

The multiplicative property of a conjugate of CTrIFN also does not hold, that is,

$(A+i B) \cdot(A-i B) \neq A^{2}-B^{2}$.

Proof. $(A+i B) .(A-i B)=A^{2}-i A B+i A B+B^{2}=\left(A^{2}+B^{2}\right)+i(A B-A B)$

$\neq A^{2}-B^{2}$ (because of the lack of additive inverse).

\section{Numerical example}

Consider the two CTrIFNs $Z_{1}=A+i B$ and $Z_{2}=C+i D$ such that,

$Z_{1}=[3,4,5,6 ; 2,4,5,7]+i[4,5,6,7 ; 3,5,6,8], Z_{2}=[2,3,4,5 ; 1,3,4,6]+i[3,4,6,8 ; 2,4,6,9]$

\section{(i) Addition}

$Z_{1}+Z_{2}=[5,7,9,11 ; 3,7,9,13]+i[7,9,12,15 ; 5,9,12,17]$

with

$$
\begin{gathered}
\mu_{A+C}(x)= \begin{cases}\frac{x-5}{2} & 5 \leq x \leq 7 \\
1 & 7 \leq x \leq 9 \\
\frac{11-x}{3} & 9 \leq x \leq 11 \\
0 & \text { otherwise }\end{cases} \\
\mu_{B+D}(x)= \begin{cases}\frac{x-7}{2} & 7 \leq x \leq 9 \\
\frac{1}{15-x} & 9 \leq x \leq 12 \\
\frac{12}{3} & \text { otherwise }\end{cases}
\end{gathered}
$$

(ii) Subtraction of CTrIFNs

$Z_{1}-Z_{2}=[-2,0,2,4 ;-4,0,2,6]+i[-4,-1,2,4 ;-6,-1,2,6]$ with

$$
\mu_{A-C}(x)=\left\{\begin{array}{ll}
\frac{x+2}{2} & -2 \leq x \leq 0 \\
1 & 0 \leq x \leq 2 \\
\frac{4-x}{2} & 2 \leq x \leq 4 \\
0 & \text { otherwise }
\end{array} \quad \nu_{A-C}(x)= \begin{cases}\frac{8-x}{4} & -4 \leq x \leq 0 \\
0 & 0 \leq x \leq 2 \\
\frac{x-2}{4} & 2 \leq x \leq 6 \\
1 & \text { otherwise }\end{cases}\right.
$$

Similarly, 


$$
\mu_{B-D}(x)=\left\{\begin{array}{ll}
\frac{x+4}{3} & -4 \leq x \leq-1 \\
1 & -1 \leq x \leq 2 \\
\frac{x+4}{3} & 2 \leq x \leq 4 \\
0 & \text { otherwise }
\end{array} \quad, \quad \nu_{B-D}(x)= \begin{cases}\frac{-x-1}{5} & -6 \leq x \leq-1 \\
0 & -1 \leq x \leq 2 \\
\frac{x-2}{5} & 2 \leq x \leq 6 \\
1 & \text { otherwise }\end{cases}\right.
$$

(iii) Product of CTrIFNs

$Z_{1} \cdot Z_{2}=[-50,-24,0,18 ;-70,-24,0,36]+i[17,31,54,83 ; 7,31,54,111]$.

(iv) Negation of CTrIFN

$-Z=[-6,-5,-4,-3 ;-7,-5,-4,-2]+i[-7,-6,-5,-4 ;-8,-6,-5,-3]$.

(v) Conjugate of a CTrIFN

$\bar{Z}=[3,4,5,6 ; 2,4,5,7]+i[-7,-6,-5,-4 ;-8,-6,-5,-3]$.

(vi) Scalar multiplication

For $k=3, k Z=[9,12,15,18 ; 6,12,15,21]+i[12,15,18,21 ; 9,15,18,24]$.

For $k=-3,-k Z=[-18,-15,-12,-9 ;-21,-15,-12,-6]$

$$
+i[-21,-18,-15,-12 ;-24,-18,-15,-9] \text {. }
$$

\section{Conclusion}

In this paper, an attempt has been made to introduce Complex Trapezoidal Intuitionistic Fuzzy Numbers (CTrIFNs). Further, few properties are discussed and verified with suitable numerical examples. It is proposed to work on establishing few more properties of CTrIFNs and their applications in quantum mechanics, electrical engineering and fluid dynamics.

\section{References}

[1] Atanassov, K. T. (1999). Intuitionistic Fuzzy Sets: Theory and Applications, Physica-Verlag, Heidelberg.

[2] Parvathi, R., \& Malathi, C. (2012). Arithmetic operations on symmetric trapezoidal intuitionistic fuzzy numbers, International Journal of Soft Computing and Engineering, 2(2), 268-273.

[3] Buckley, J. J. (1989). Fuzzy complex numbers, Fuzzy Sets and Systems, 33, 333-345.

[4] Beaula, T., \& Priyadharsini, M. (2015). Operations on intuitionistic trapezoidal fuzzy numbers using interval arithmetic, Int. J. of Fuzzy Mathematical Archive, 9(1), 125-133.

[5] Moore, R. E. (1996). Interval Analysis, Prentice Hall, India.

[6] Burillo, P., Bustince, H., \& Mohedano, V. (1994). Some definition of intuitionistic fuzzy number, Fuzzy Based Expert Systems, Sofia, Bulgaria, 1994, 28-30. 